\title{
LA NEOFOBIA ALIMENTARIA COMO INSTRUMENTO PARA LA SEGMENTACIÓN DE CONSUMIDORES DE VINO
}

\author{
$\underline{\text { Adrián Rabadán }}^{a *}$, Mónica Díaz $^{b}$ y Rodolfo Bernabéu ${ }^{a}$
}

a Escuela Técnica Superior de Ingenieros Agrónomos y de Montes, Universidad de Castilla-La Mancha (Albacete, Adrian.Rabadan@uclm.es;Rodolfo.Bernabeu@uclm.es) ${ }^{b}$ Escuela Técnica Superior de Ingenieros Agrónomos, Universidad de Castilla-La Mancha (Ciudad Real, Monica.Diaz@uclm.es )

\begin{abstract}
Resumen
La innovación en el sector agroalimentario es un elemento clave para garantizar la producción de alimentos más sanos, nutritivos y sostenibles. Sin embargo, existen consumidores que se muestran reacios a aceptar estas innovaciones, especialmente cuando afectan a productos más tradicionales como es el caso del vino. Este trabajo analiza el nivel de neofobia de los consumidores de vino en España, evaluando tanto el rechazo que existe a probar nuevos vinos, como el rechazo que muestran hacia la utilización de nuevas tecnologías en la producción de alimentos. Los resultados demuestran que no existe una correlación directa entre estos dos tipos de neofobia. Mediante un clúster jerárquico, se han identificado cuatro segmentos diferentes de consumidores de vino, con diferentes percepciones frente a los dos tipos de neofobia analizados. El segmento de consumidores que muestra una mayor predisposición hacia la aceptación de nuevos vinos obtenidos, además, utilizando nuevas tecnologías, es el formado por los consumidores que presentan una mayor frecuencia de consumo, rentas más altas y un mayor nivel de estudios.
\end{abstract}

Palabras Clave: Innovación, nuevos vinos, nuevas tecnologías de producción.

\section{Introducción}

Actualmente la innovación se considera un elemento básico para mejorar la competitividad y la rentabilidad de las producciones agroalimentarias. Sin embargo, el desarrollo de alimentos novedosos se encuentra con la oposición de algunos segmentos de consumidores que perciben riesgos o desventajas en estos productos innovadores. Esta reticencia a la aceptación y/o consumo de nuevos alimentos se ha denominado neofobia alimentaria (Henriques et al., 2009). En los últimos treinta años se han desarrollado diferentes instrumentos destinados a medir la neofobia alimentaria de los consumidores, destacando entre todos ellos la Food Neophobia Scale (FNS) (Pliner y Hobben, 1992). Tras el éxito de la FNS se han desarrollado otras herramientas para medir aspectos más específicos de la neofobia, como la Food Technology Neophobia Scale (FTNS), desarrollada específicamente para evaluar la predisposición de los consumidores a aceptar los alimentos producidos utilizando nuevas tecnologías (Cox y Evans, 2008).

La innovación de producto y de proceso se ha ido imponiendo incluso en los sectores agroalimentarios ligados de forma más intensa a una producción más tradicional, como es el caso del sector del vino. Al tratarse de un producto con unas características muy específicas, para evaluar la neofobia de los consumidores a probar nuevos vinos (WN), se ha desarrollado una nueva escala: la Wine Neophobia Scale (Ristic et al., 2016). Diversos estudios han identificado que los consumidores de diferentes países muestran niveles diferentes de WN (Nguyen et al., 2019). En este sentido es de destacar que, a pesar de la importancia del sector del vino en España, es difícil encontrar información relevante sobre el nivel de WN de los consumidores de vino españoles.

En el escenario actual de creciente utilización de la innovación en el sector del vino, se considera crucial identificar si existen consumidores dispuestos a adquirir nuevos vinos y vinos producidos utilizando nuevas tecnologías. Además, es necesario identificar qué valoran estos consumidores que muestran actitudes más favorables a la adquisición de estos vinos innovadores y cuál es su perfil sociodemográfico. Solo a partir de esta información se podrán diseñar y desarrollar nuevos vinos adaptados, de forma específica, a las preferencias de sus consumidores potenciales, garantizando así su éxito comercial.

\section{Materiales y métodos}

\subsection{Base de datos}

Las encuestas fueron realizadas mediante una entrevista personal en los alrededores de establecimientos comerciales a consumidores. Concretamente, la encuesta se realizó en el área metropolitana de Madrid en septiembre de 2019. Un total de 400 consumidores de vino fueron entrevistados. El máximo error de muestreo fue inferior al 5,0\% para un intervalo de confianza del 95,5\% $(\mathrm{k}=2)$ bajo el principio de máxima indeterminación $(\mathrm{p}=\mathrm{q}=50 \%)$. 


\subsection{Metodología}

El cuestionario incluía la evaluación de varias escalas especialmente diseñadas para evaluar la neofobia de producto y proceso de los consumidores de vino. Para evaluar la neofobia a probar nuevos vinos se utilizó la WNS. Para ello, durante la entrevista, se pidió a los consumidores que valoraran su grado de acuerdo para cada uno de los ítems de la WNS utilizando una escala Likert de 9 puntos. El valor final de la WNS para cada consumidor se realizó sumando las puntuaciones de cada uno de los ítems. Así, una puntuación final elevada indicaba que el consumidor presentaba unos niveles elevados de neofobia, indicando una menor tendencia a probar nuevos vinos o vinos innovadores. Por el contrario, las puntuaciones más bajas indicaban que el consumidor presentaba una elevada predisposición a probar nuevos vinos. Para evaluar la neofobia de los consumidores de vino hacia la aplicación de nuevas tecnologías en la producción de alimentos se utilizó la Abbreviated Food Technology Neophobia Scale (AFTNS) basada en la escala original de Cox y Evans (2008). En esta escala, los consumidores valoraron su grado de acuerdo para cada uno de los 9 ítems de la escala utilizando una escala Likert de 6 puntos.

Adicionalmente, los consumidores indicaron su frecuencia de consumo de vino y valoraron, utilizando una escala Likert de 5 puntos, la importancia que atribuían a diferentes atributos del vino en el momento de su adquisición. Finalmente, para incluir el análisis de las variables demográficas y socioeconómicas, a los consumidores se les pidió que respondieran varias preguntas que incluían información de su género, edad, máximo nivel de educación completado y renta familiar mensual.

Para analizar los datos, y de forma similar a lo propuesto por Schnettler et al., (2017), se realizó un análisis clúster jerárquico con el objetivo de segmentar a los consumidores utilizando para ello las valoraciones finales obtenidas para cada consumidor en la WNS y la AFTNS (Gráfico 1). Para identificar las diferencias entre segmentos se utilizó un análisis de varianza con un Tukey HSD post-hoc (nivel de significancia del $5 \%$ ) para los atributos del vino y para las escalas y un test $\mathrm{Chi}^{2}$ de Pearson para las variables discretas. El estudio estadístico se realizó con el Paquete Estadístico para Ciencias Sociales IBM SPSS versión 23.

Gráfico 1. Esquema de trabajo utilizado para la segmentación de los consumidores

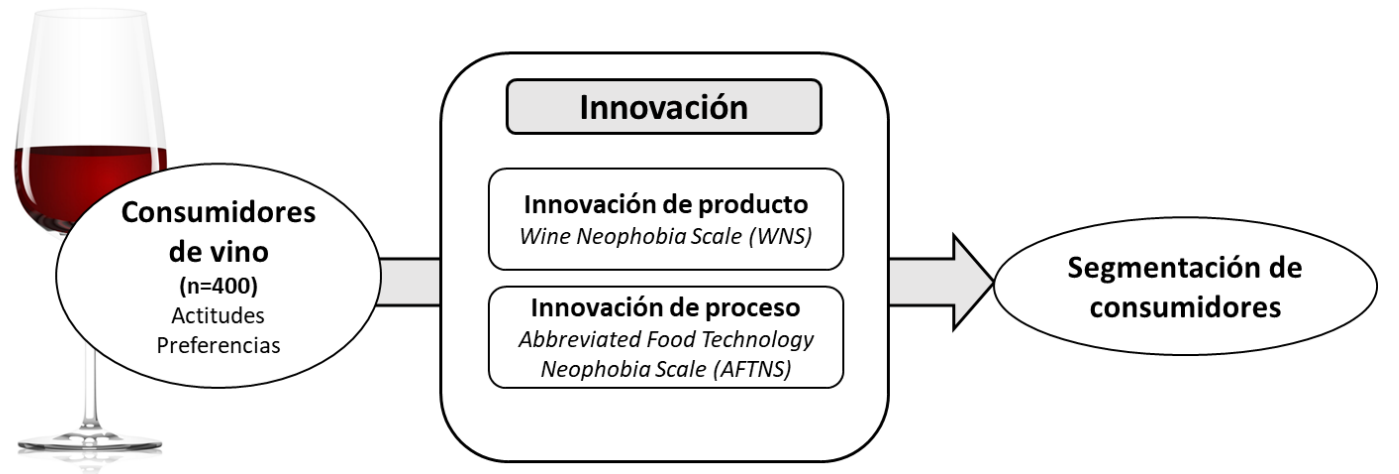

\section{Resultados}

Los resultados obtenidos mostraron que no existía una relación significativa entre el rechazo a probar nuevos vinos y el rechazo hacia los productos producidos utilizando tecnologías novedosas. Por tanto, estos tipos de neofobia (WN y FTN) servirían para evaluar diferentes aspectos de la neofobia alimentaria. El resultado de la segmentación de consumidores atendiendo a los valores obtenidos en la WNS y en la AFTNS se muestra en el Cuadro 1.

Los consumidores que presentan un nivel de WN más baja, son aquellos consumidores que tienen mayor frecuencia de consumo (consumo mínimo de una vez a la semana). Así, una mayor exposición al producto parecería estar relacionada con una mayor predisposición a probar nuevos vinos. Esa mayor frecuencia de consumo, y el mayor conocimiento del producto ligado a ese mayor consumo, hace que estos consumidores sean también los que dan valoraciones más altas a la mayoría de los atributos del vino. Estos consumidores más implicados valoran en mayor medida atributos como el tipo de vino (blanco, tinto o rosado) o la existencia de crianza en el proceso de producción.

Es destacable también la escasa valoración que todos los grupos de consumidores hacen del atributo "ecológico", que obtiene en todos los casos las valoraciones más bajas, quedando muy por detrás de la importancia atribuida a otras figuras de calidad diferenciada como las Indicaciones Geográficas. La valoración es, sin embargo, significativamente más alta entre los segmentos de consumidores con una WN 
más baja. Por tanto, a pesar de no ser un atributo relevante para ningunos de los segmentos identificados, los consumidores más neófilos son, en general, los que en mayor medida valoran el atributo vino ecológico.

Cuadro 1. Segmentación de consumidores utilizando los resultados de la Wine Neophobia Scale (WNS) y la Abbreviated Food Technology Scale (AFTNS).

\begin{tabular}{|c|c|c|c|c|}
\hline & $\begin{array}{c}\text { Cluster } 1 \\
\text { Neófilos } \\
(25,5 \%)\end{array}$ & $\begin{array}{l}\text { Cluster } 2 \\
\text { Neófilos } \\
\text { anti-NT } \\
(23,3 \%) \\
\end{array}$ & $\begin{array}{c}\text { Cluster } 3 \\
\text { Neófobos pro- } \\
\text { NT } \\
(28,0 \%) \\
\end{array}$ & $\begin{array}{c}\text { Cluster } 4 \\
\text { Neófobos } \\
(23,3 \%)\end{array}$ \\
\hline \multicolumn{5}{|l|}{ Escalas } \\
\hline WNS & $23,59 b$ & $24,06 b$ & $40,34 a$ & $41,33 \mathrm{a}$ \\
\hline AFTNS & $18,79 \mathrm{c}$ & $33,25 \mathrm{a}$ & $21,45 b$ & $34,49 a$ \\
\hline \multicolumn{5}{|l|}{ Frecuencia de consumo $(\%)$} \\
\hline Varias veces a la semana & 20,6 & 21,5 & 9,8 & 18,3 \\
\hline $\begin{array}{l}\text { Al menos una vez por } \\
\text { semana }\end{array}$ & 43,1 & 40,9 & 18,8 & 20,4 \\
\hline Cada dos semanas & 8,8 & 15,1 & 15,2 & 15,1 \\
\hline Una vez al mes o menos & 27,5 & 22,6 & 26,3 & 46,2 \\
\hline \multicolumn{5}{|l|}{ Atributos del vino } \\
\hline Tipo & $4,32 \mathrm{ab}$ & $4,59 \mathrm{a}$ & $4,15 b$ & $4,48 \mathrm{a}$ \\
\hline Variedad & $3,53 \mathrm{a}$ & $3,66 \mathrm{a}$ & $2,71 b$ & $3,01 \mathrm{~b}$ \\
\hline Crianza & $4,09 \mathrm{a}$ & $4,01 \mathrm{a}$ & $2,95 b$ & $3,31 b$ \\
\hline Precio & 3,77 & 4,22 & 3,95 & 4,12 \\
\hline Origen & $3,63 \mathrm{ab}$ & $3,88 \mathrm{a}$ & $3,31 \mathrm{c}$ & $3,32 \mathrm{bc}$ \\
\hline Marca/Bodega & $3,94 \mathrm{a}$ & $3,90 \mathrm{a}$ & $3,27 b$ & $3,56 a b$ \\
\hline Estética & 3,16 & 3,30 & 2,71 & 2,85 \\
\hline Indicación geográfica & $3,69 \mathrm{a}$ & $3,67 \mathrm{a}$ & $3,04 b$ & $3,25 b$ \\
\hline Ecológico & $2,36 \mathrm{a}$ & $2,45 \mathrm{a}$ & $1,94 b$ & $1,75 b$ \\
\hline \multicolumn{5}{|l|}{ Género $(\%)$} \\
\hline Hombre & 65,7 & 57,0 & 50,0 & 55,9 \\
\hline Mujer & 34,3 & 43,0 & 50,0 & 44,1 \\
\hline \multicolumn{5}{|l|}{$\operatorname{Edad}(\%)$} \\
\hline $18-24$ & 11,8 & 11,8 & 17,0 & 15,1 \\
\hline $25-34$ & 20,6 & 23,7 & 20,5 & 19,4 \\
\hline $35-46$ & 25,5 & 22,6 & 17,0 & 15,1 \\
\hline $50-64$ & 28,4 & 23,7 & 24,1 & 23,7 \\
\hline $65+$ & 13,7 & 18,3 & 21,4 & 26,9 \\
\hline \multicolumn{5}{|l|}{ Estudios (\%) } \\
\hline Primaria & 20,6 & 29,0 & 28,6 & 36,6 \\
\hline Secundaria & 41,2 & 35,5 & 48,2 & 31,2 \\
\hline Universidad & 34,3 & 33,3 & 23,2 & 32,3 \\
\hline Postgrado & 3,9 & 2,2 & 0,0 & 0,0 \\
\hline \multicolumn{5}{|l|}{ Renta $(\%)$} \\
\hline$<1500 €$ & 9,3 & 33,8 & 7,2 & 21,9 \\
\hline $15000-2100 €$ & 13,5 & 9,3 & 38,1 & 29,3 \\
\hline $2101-3000 €$ & 39,6 & 32,6 & 41,2 & 28,0 \\
\hline$>3000 €$ & 37,5 & 24,4 & 13,4 & 20,7 \\
\hline
\end{tabular}

Los valores de la Chi Cuadrado para la frecuencia de consumo y las variables socioeconómicas son: frecuencia de consumo, $X^{2}=44.101, \mathrm{df}=9, \mathrm{p}=0.000$; género, $X^{2}=5.42, \mathrm{df}=3, \mathrm{p}=0.143$; edad, $\mathrm{X}^{2}=26.79, \mathrm{df}=12, \mathrm{p}=0.571$; educación, $X^{2}=18.703$, df $=9, p=0.028 ;$ renta, $X^{2}=72.580$, df $=12, p=0.000$.

Respecto a las características socioeconómicas, no se han identificado diferencias significativas entre los grupos identificados atendiendo a la neofobia alimentaria. Sin embargo, sí se observa que los consumidores con las rentas más altas son aquellos que muestran unos niveles de neofobia más bajos, tanto hacia nuevos vinos como hacia nuevas tecnologías aplicadas a la producción de alimentos. Además, estos consumidores 
son los que menor importancia dan al precio, aunque no se observan diferencias significativas con los otros grupos.

\section{Conclusiones}

Este estudio demuestra que los segmentos de consumidores de vino identificados muestran diferencias significativas en su predisposición a probar nuevos vinos o en su predisposición a adquirir alimentos elaborados utilizando nuevas tecnologías. Atendiendo a los resultados obtenidos, el desarrollo de vinos innovadores debería estar dirigido a un segmento de consumidores muy implicado con el producto (alta frecuencia de consumo) y que presenta mayores rentas y un nivel educativo más alto. En el diseño y comercialización de estos vinos novedosos habría que prestar especial atención a los atributos tipo de vino, crianza, y marca o bodega productora, ya que son estos los atributos que en mayor medida influyen en la decisión de compra del segmento de consumidores que mayor predisposición de compra muestra por estos productos.

\section{Agradecimientos}

Este estudio ha sido financiado por la Diputación de Albacete, la Universidad de Castilla-La Mancha y el Fondo Europeo de Desarrollo Regional [2018/11744].

\section{Bibliografía}

Cox, D.N. y Evans, G. (2008). 'Construction and validation of a psychometric scale to measure consumers' fears of novel food technologies: The food technology neophobia scale". Food Quality and Preference, 19(8): 704-710.

Henriques, A.S., King, S.C. y Meiselman, H.L. (2009). "Consumer segmentation based on food neophobia and its application to product development”. Food Quality and Preference, 20(2): 83-91.

Pliner, P., Hobden, K. (1992). "Development of a scale to measure the trait of food neophobia in humans". Appetite, 19(2): 105-120.

Ristic, R., Johnson, T.E., Meiselman, H.L. y Hoek, A.C., Bastian, S.E.P. (2016). "Towards development of a Wine Neophobia Scale (WNS): Measuring consumer wine neophobia using an adaptation of The Food Neophobia Scale (FNS)". Food Quality and Preference, 49: 161-167

Schnettler, B., Grunert, K.G., Miranda-Zapata, E., Orellana, L., Sepúlveda, J., Lobos, G., Hueche, C. y Höger, Y. (2017). "Testing the Abbreviated Food Technology Neophobia Scale and its relation to satisfaction with food-related life in university students". Food Research International 96: 198-205. 